\title{
Ultrasound-Based Indoor Robot Localization Using Ambient Temperature Compensation
}

\author{
Csaba NAGY, Zalán BIRÓ-AMBRUS, Lőrinc MÁRTON \\ Department of Electrical Engineering, Faculty of Technical and Human Sciences, \\ Sapientia Hungarian University of Transylvania, Tg. Mureş, Romania \\ e-mail: csaba3nagy@yahoo.com, birozali@yahoo.com, martonl@ms.sapientia.ro
}

Manuscript received January 24, 2016; revised September 15, 2016

\begin{abstract}
This paper focuses on the hardware development of an indoor ultrasound based robot localization system. The problems related to the ultrasound based distance measurements are presented and solutions are proposed related to Time of Flight measurements and measurement synchronization. An optimization based compensation method is introduced to attenuate the effect of the ambient temperature on the distance measurement precision. Experimental measurements were performed to analyze the applicability of the developed system and measurement methods.
\end{abstract}

Keywords: robot tracking, ultrasound, mobile robot, localization, distance measurement.

\section{Introduction}

Robot positioning and tracking is an important issue when developing complex applications in the fields of robotics. The position measurement part of the mobile robot control system should provide accurate information and high measurement rate when tracking control algorithms are executed with high speeds. Generally, the robots are equipped with dead reckoning sensors such as inertial measurement units and encoders. Those are supplying the robot's position with high update rate but the measurement cumulates error over time, it becomes inaccurate. The solution is to use a secondary position measurement source with lower update rate and reduce the cumulated error. For outdoor applications GPS is suitable to provide secondary position data for mobile robots and flying drones. However, the GPS can hardly be used for indoor localization.

For indoor localization multiple solutions can be applied. The work floor can be covered with RFID (Radio Frequency Identification) tags and a tag reader mounted to the robot. This method provides a precision in the order of centimeters [1]. Other solutions try to locate the robot based on Bluetooth [2] or on Wi-Fi [3] signal strength. This method offers a precision of tens of centimeters and the update rate is not suitable for robot control. 
Other common method is the ultrasound based time of flight measurement. In the work [4] a setup consisting of 1 transmitter and 2 receivers is used to position a mobile robot. The transmitter uses radio frequency signals to send the reference for the measurement. The measuring error is corrected using a Linear Kalman Filter. Using this technique a maximum error of $2 \mathrm{~cm}$ can be achieved in a $3.5 \mathrm{~m} \times 2.2 \mathrm{~m}$ working environment. The paper [5] presents a method to combine a RF and ultrasound based wireless positioning with the measurements of dead-reckoning sensors using Unscented Kalman Filter.

Our goal is to design and implement an ultrasound time of flight based localization system with multiple robot control capability. In our system the ultrasonic transmitters are located on the robots, and they are synchronized to the receivers with infrared signals, and ceiling mounted ultrasonic receivers interconnected with RS485 bus to the control computer. The measured distances can be processed using 2D or 3D localization algorithms and transmitted to the robots where the position values are used. This study is an extended version of the conference paper [10].

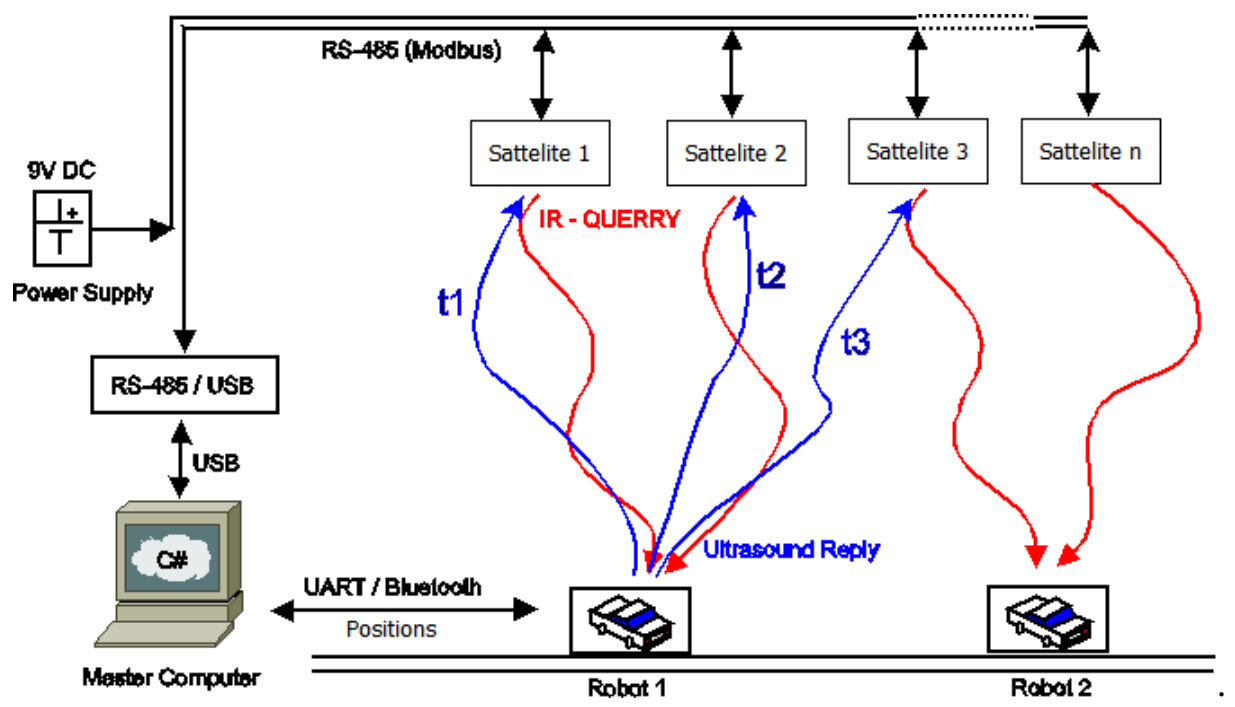

Figure 1: The block diagram of the proposed system

\section{Ultrasound-based positioning systems}

\subsection{General system description}

The block diagram of the localization system is shown in Fig. 1. The measurement is initiated by the master computer, which configures the next robot ID and broadcasts a synchronization command for all the anchors through the RS485 bus. The controllers of the anchors receive this command through their interrupt inputs and transmit an infrared (IR) packet containing the robot ID for the ultrasonic receiver node placed on the robot. If the node recognizes 
his own ID, it responds with a phase-modulated ultrasonic (US) chirp signal [6]. Simultaneously the controllers of the anchors start to sample the ultrasonic receiver. The phase change detection a offers superior precision of the arrival time instant. The ultrasonic signal arrives at the anchor's receiver with Time-ofFlight plus the constant delay between the received IR signal and the transmitted ultrasonic response added by the beacon. After the measurement is finished the Master computer queries the measured times from each node.

\subsection{Time of flight measurement}

We implemented a distance measurement method based on amplitude and phase modulation similarly as in [6], see Fig. 2. The method reduces the error induced by the pendulum effect at the transmitter and the receiver, by introducing a phase modulation in the middle of the measurement signal. The theoretical precision achievable by this method is $0.1 \%$ of the wavelength.

In the case of the classic Time of Flight (ToF) measurement the time is calculated until the first instance when the received signal reaches a certain threshold. Because of high inertia of the ultrasound sensor, the resonating time wearies with the distance, and the measurement error can be 2-3 wavelengths long which correspond to a couple of centimeters.

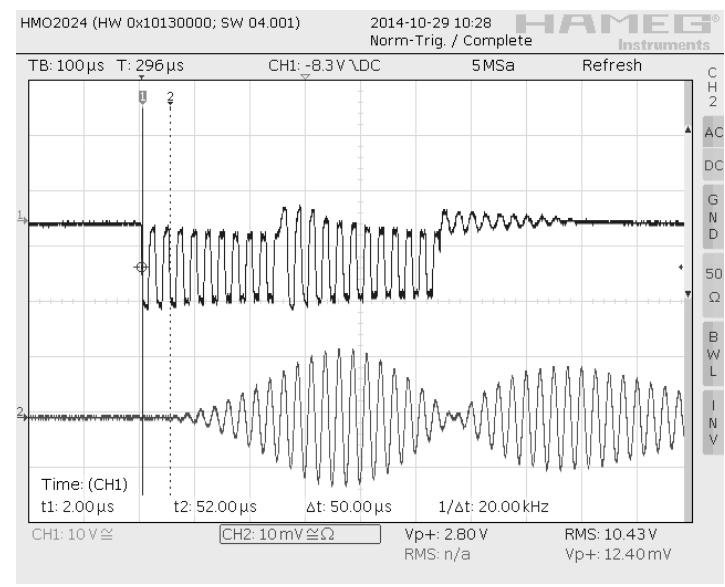

Figure 2: The transmitted and received waveform

Ultrasonic trans-receiver is constructed with a low jitter Infrared receiver [7], a wide angle ultrasonic transmitter [8] along with an amplifier and a dsPIC microcontroller. The task of the trans-receiver is to monitor for the infrared packets, synchronize for the packet start and, if the packet matches the ID, a modulated ultrasonic pulse is transmitted. 


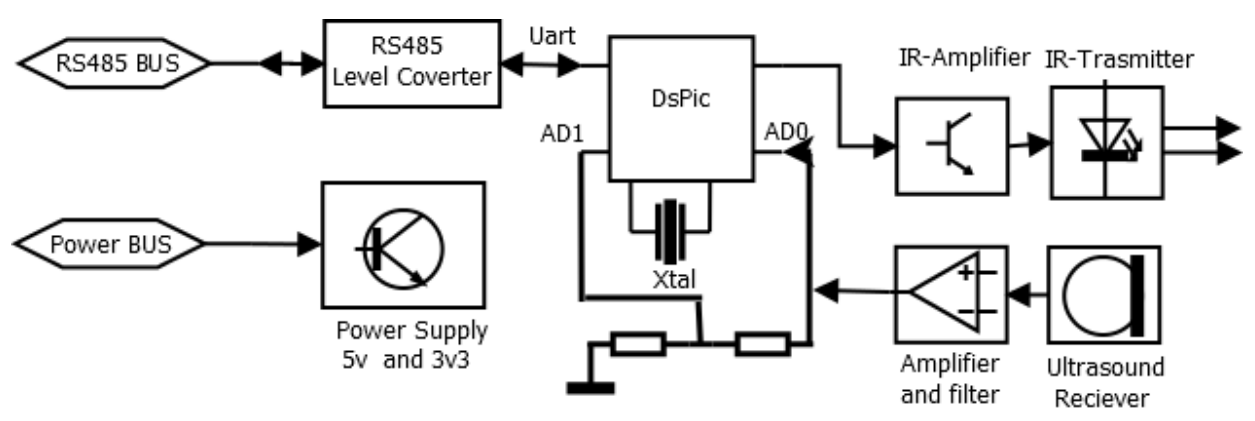

Figure 3: Block diagram of the anchor

The anchor contains a power supply, a high power infrared LED-s, an ultrasonic receiver, combined with a $40 \mathrm{kHz}$ band pass filter (see Fig. 3). The received signal is sampled simultaneously by two AD inputs of the microcontroller at $1.2 \mathrm{Ms} / \mathrm{s}$ speed. One of the inputs expects signals with lower amplitude the other expects signals with higher amplitude. When the data is analyzed, if the higher amplitude inputs are clipping, then the lower amplitude input is used. The reference for the $\mathrm{AD}$ converter is $3.3 \mathrm{~V}$ and, because the amplifier is powered from $5 \mathrm{~V}$, the maximum input voltage can be up to $5 \mathrm{~V}$.This solution increases the precision when the received signal has small amplitude, but keeps working properly at the minimal distance even if the amplified signal is over $5 \mathrm{~V}$. Because the signal is not affected by separate amplifying stages, the phases of the two signals are exactly the same.

\subsection{Robot position computation}

In order to calculate the robot's position simple calculations are performed as shown in Fig. 4.Denote with $d 1, d 2, d 3$ and $d 4$ are the distances measured by the anchors, with each anchor placed in the same $h$ height and with a distance $D$ between the two anchors employed.

$d i$ is computed as the product of the ultrasound propagation speed and the measured time of flight.

The $\left(X_{p}, Y_{p}\right)$ coordinates of the robot can be calculated from equations given in (5) and (6):

$$
\begin{gathered}
\left\{\begin{array}{c}
X p^{2}+Y p^{2}=s 1^{2} \\
X p^{2}+(D-Y p)^{2}=s 2^{2}
\end{array}\right. \\
s 1=\sqrt{d_{1}{ }^{2}-h^{2}}, s 2=\sqrt{d_{2}{ }^{2}-h^{2}}
\end{gathered}
$$




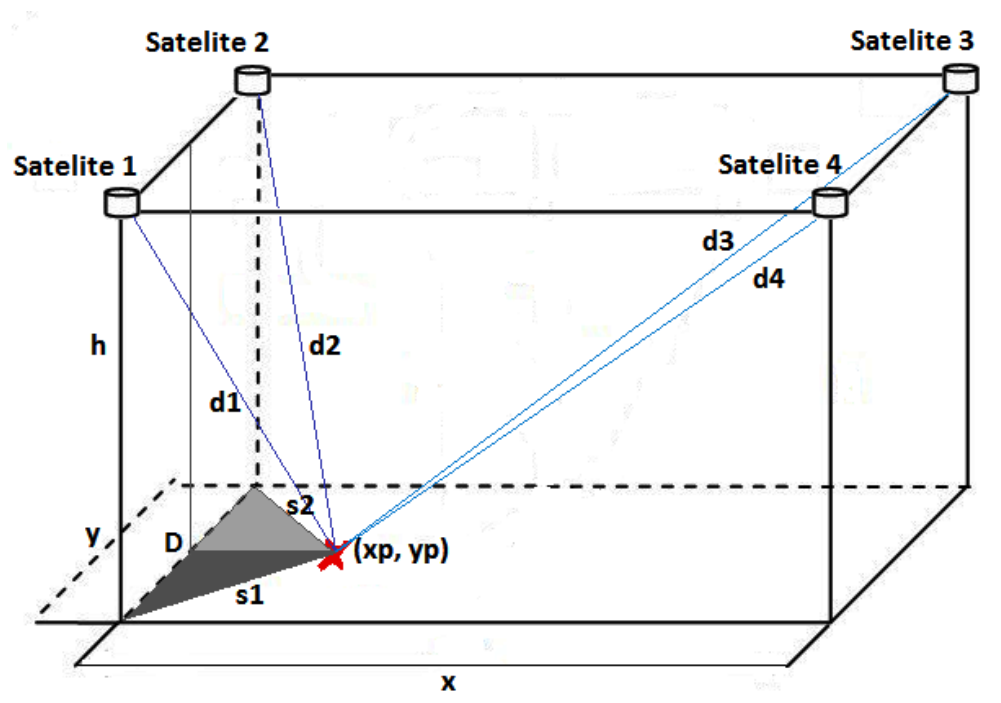

Figure 4: The robot positioning based on the measured distances

\subsection{Online temperature approximation and compensation}

When the time of flight measurement is available, the distance between the anchor and the robot can be computed using the speed of the ultrasound. The speed of sound varies in function of the environment temperature. The effect of the temperature change is the following formula [5]:

$$
c=331.3+0.6 \cdot t_{\text {ambient }}
$$

where $c$ is the propagation speed of the ultrasound signal and $t_{\text {ambient }}$ is the temperature of the sensor's environment. Based on the equation (1) we can conclude that in the case of high ambient temperature the sound travels at higher speeds and the measured distance will be smaller than the real distance, i.e. the calculated position will also drift from the real. Using more measurement pairs, from different anchors and minimizing the distance between the measurement results, we can compute the environment temperature, using which the temperature can be calibrated. The criteria function which is used during the calibration is defined as:

$$
J^{T}=\sum_{i=1}^{n-1} \sum_{j=i+1}^{n} \operatorname{dist}\left(\left(\begin{array}{l}
X_{i}^{T} \\
Y_{i}^{T}
\end{array}\right),\left(\begin{array}{l}
X_{j}^{T} \\
Y_{j}^{T}
\end{array}\right)\right)^{2}
$$


where $n$ is the number of anchor pairs used for distance measurements and $\left(\begin{array}{l}X_{i}^{T} \\ Y_{i}^{T}\end{array}\right)$ denotes the calculated position for the $i$-th pair at temperature $T$.

During the distance measurements we modify the $t_{a c t}$ estimated temperature using the following approach: let $t_{1}=t_{a c t}+0.1$ and $t_{2}=t_{a c t}-0.1$.

$$
\begin{aligned}
& \text { if }\left(J_{t 1}<J_{\text {tact }}\right) \text { thent } t_{a c t}=t_{1} \\
& \text { if }\left(J_{t 2}<J_{\text {tact }}\right) \text { thent } t_{a c t}=t_{2}
\end{aligned}
$$

With the temperature computed as above, the real ultrasound speed can be computed on-line.

Remark: The proposed method works when multiple measurements are available because the method uses the difference between the multiple results.

\section{Experimental measurements}

The installed system uses 4 anchors and one robot. The anchors are installed at height of $2.9 \mathrm{~m}$ and the work area is $3 \mathrm{~m} \times 3 \mathrm{~m}$. The trans-receiver is mounted on the KUKAyouBot at the height $0.8 \mathrm{~m}$. The robot's position is calculated using 2 distances and the two-dimension localization described above hence the robot moves in a horizontal plane. The position is calculated for each pair of anchors and the result is averaged. The achieved precision is under $1 \mathrm{~cm}$ when all the anchors are visible and the update rate is $8 \mathrm{~Hz}$. The anchors were calibrated using a laser distance measurement tool having $2 \mathrm{~mm}$ precision. 


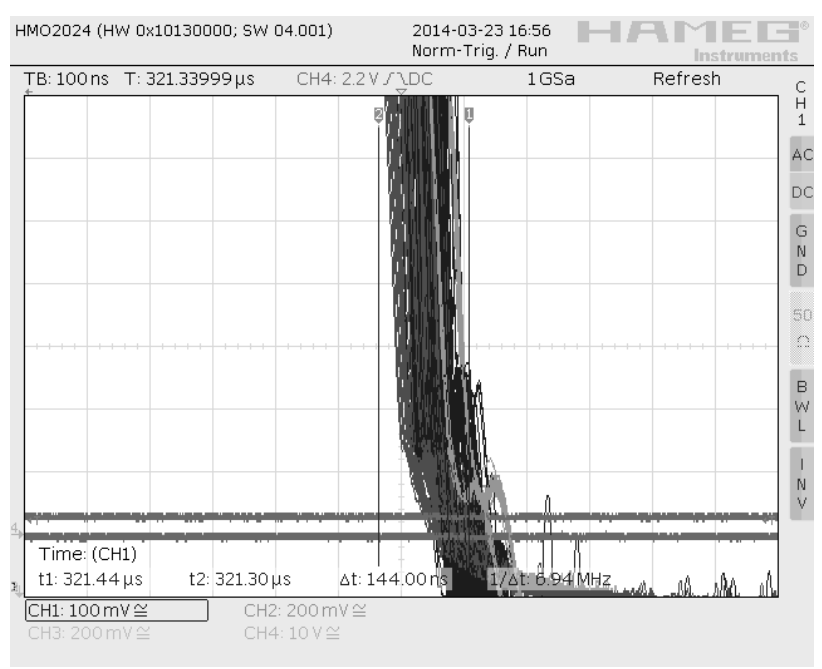

Figure 5: The synchronization jitter over the R485 bus

The synchronization jitter was measured using a digital oscilloscope in persistence mode and multiple measuring cycles were performed. Four ultrasound receivers are connected to the four inputs of the oscilloscope, see Fig. 5. The worst case synchronization error due to the interrupt handling is under 144 ns.

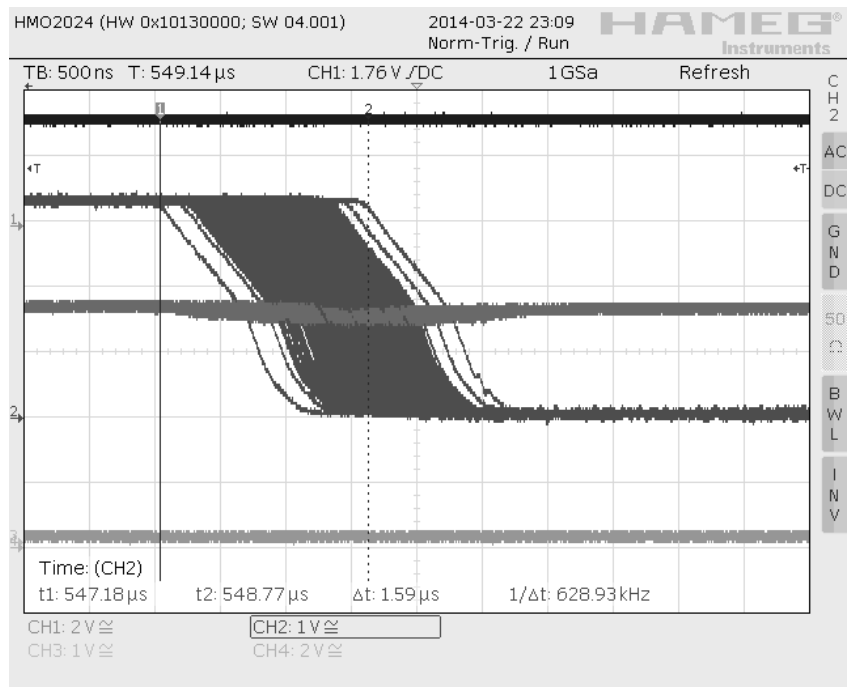

Figure 6: The synchronization jitter on the infrared link

The synchronization jitter on the infrared link is dependent on many variables such as background illumination, distance from the transmitter, but in our case the synchronization jitter, using the same method is under $1.6 \mu$ s (see Fig. 6), approximately $0.6 \mathrm{~mm}$ in distance. If the ambient light is too powerful or 
the received signal is too small, the jitter can jump up to $40 \mu$ s equal to $4.6 \mathrm{~mm}$ in the distance measurement. To maximize the received light power, all satellites transmit simultaneously the infrared code.

The cost function based temperature estimation method is shown in Fig.7. As it can be seen, the figure algorithm estimates well the ambient temperature in the vicinity of the ultrasound sensor, using only distance measurements. As a results the distance measurement offset can be considerably reduced.
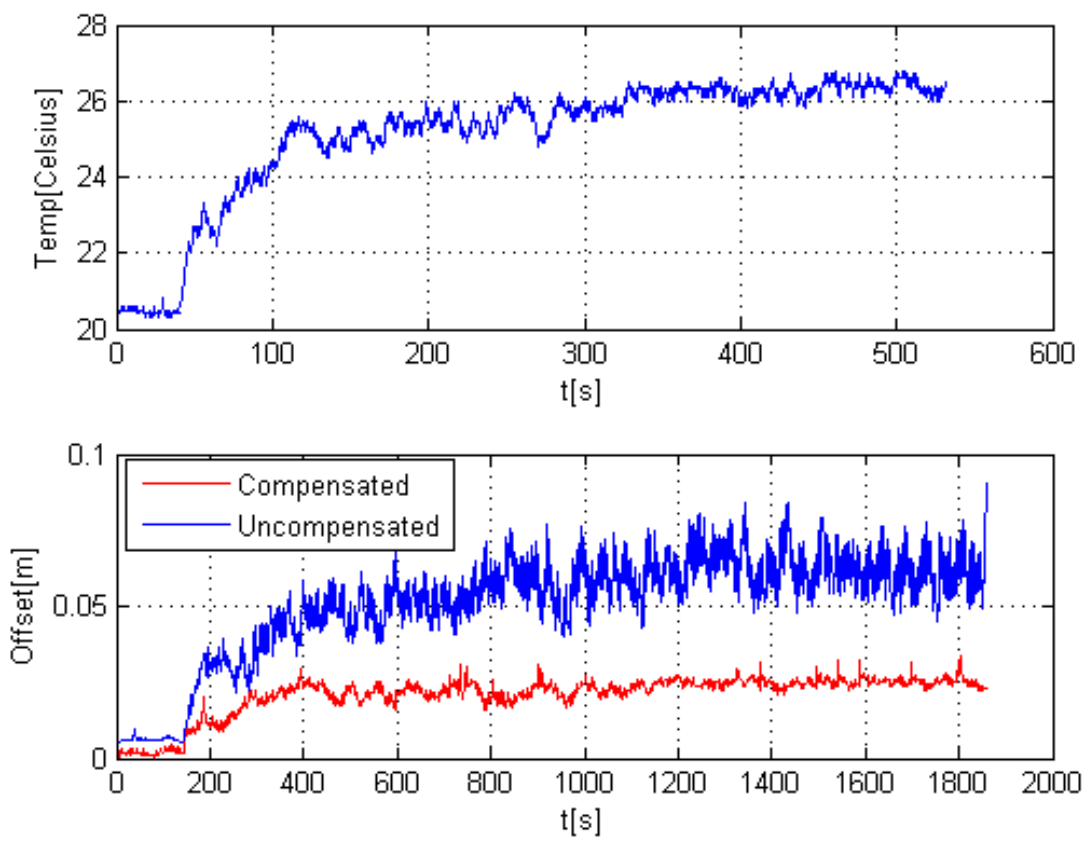

Figure 7: Comparison of the localization error between the compensated and the uncompensated position error while the temperature is changing

Fig. 8 shows the localization of a KUKA youBot mobile platform, on which the ultrasonic receiver is mounted. The robot's reference path and the measured real path are presented in this figure. 


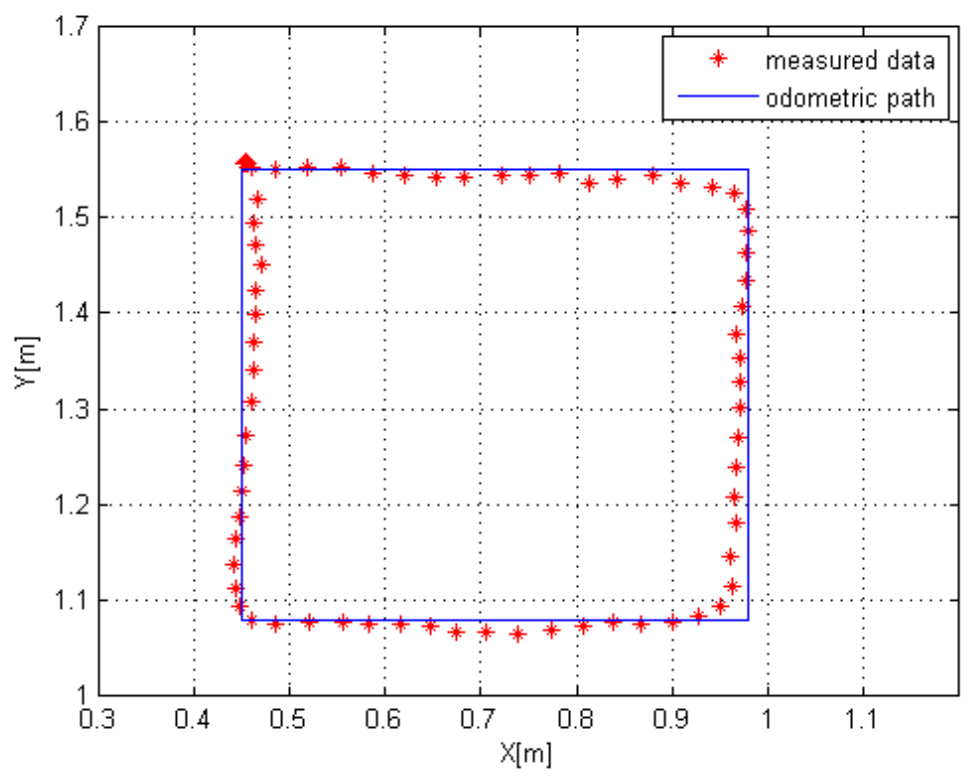

Figure 8: The path of the robot using a rectangular reference

\section{Conclusions}

We developed an ultrasound-based localization system capable of tracking multiple mobile robots in indoor environment. The proposed solution applies ultrasonic signal Time of Flight distance measurement. To obtain precise measurements in the presence of varying ambient temperature a Monte Carlo method based temperature estimation technique was proposed. From distance measurements the robot's position was computed using triangulation formulas. Based on the measurements we approximated the environment temperature. The proposed solution was successfully applied for robot position calculation in the vertical plane.

\section{Acknowledgements}

The research and publication has been supported by the European Union and Hungary and co-financed by the European Social Fund through the project TAMOP-4.2.2.C-11/1/KONV-2012-0004 - National Research Center for Development and Market Introduction of Advanced Information and Communication Technologies (subproject I.6). 


\section{References}

[1] Park, S. and Hashimoto, S., "Autonomous mobile robot navigation using passive RFID in indoor environment", IEEE Transactions on Industrial Electronics, vol. 56, no. 7, July 2009, pp. 2366-2373.

[2] Raghavan, A., Ananthapadmanaban, H., Sivamurugan, M., and Ravindran, B.,"Accurate mobile robot localization in indoor environments using bluetooth", in IEEE International Conference on Robotics and Automation (ICRA), May 2010, pp. 4391-4396.

[3] Biswas, J., and Veloso, M., "WiFi localization and navigation for autonomous indoor mobile robots", in IEEE International Conference on Robotics and Automation (ICRA), May 2010, pp. 4379-4384.

[4] Kim, D.E., Hwang, K. H., Lee, D. H., Kuc, T. J., "A Simple Ultrasonic GPS System for Indoor Mobile Robot System using Kalman Filtering”, SICE-ICASE, 2006, pp. 2915-2918.

[5] Zhang, Y., Wu, C., Cheng, L., Chu, H., "Localization and Tracking of Indoor Mobile Robot with Ultrasonic and Dead-reckoning Sensors", Journal of Computational Information Systems, 2012, vol. 8, no. 2, pp. 531-539.

[6] Huang,Y. P., Wang, J. S., Huang, K. N., Ho, C. T., Huang, J. D., and Young, M. S., "Envelope pulsed ultrasonic distance measurement system based upon amplitude modulation and phase modulation," Review of Scientific Instruments, vol. 78, no. 6, 2007.

[7] Vishay, "IR Reciever Modules for 3D Synchronization Signals",TSOP35D25.

[8] Prowave," Air Ultrasonic Ceramic Transducers", 400ET080.

[9] Boyd, S., Vandenberghe, L., “Convex Optimization”, Cambridge university press. ISBN 978-0-521-83378-3.

[10] Nagy, Cs., Biro, Z., Marton, L., "Development of an Ultrasound Based Tracking System for Indoor Robot Localization", in 5th International Conference on Recent Achievements in Mechatronics, Automation, Computer Sciences and Robotics, Tg. Mures, Romania, pp. 155-162, 2015. 